\title{
On some integral inequalities via $h$-convexity
}

\author{
Mevlüt Tunç
}




\title{
ON SOME INTEGRAL INEQUALITIES VIA $h$-CONVEXITY
}

\author{
MEVLÜT TUNÇ
}

Received 21 February, 2012

\begin{abstract}
In this paper, we establish some new inequalities for class of $S X(h, I)$ convex functions which are super-multiplicative or super-additive and nonnegative. And we also give some applications for special means.
\end{abstract}

2010 Mathematics Subject Classification: 26D15; 26A51

Keywords: Hadamard's inequality, $h$-convex, super-multiplicative, super-additive, similarly ordered

\section{INTRODUCTION}

In the sequel of the paper, $I$ and $J$ are intervals in $\mathbb{R}$, where $I$ is a convex set, $(0,1) \subseteq J$ and functions $h$ and $f$ are real non-negative functions defined on $J$ and $I$, respectively.

The following definition is well known in the mathematical literature : [20] A function $f: I \rightarrow \mathbb{R}, \varnothing \neq I \subseteq \mathbb{R}$, where $I$ is a convex set, is said to be convex on $I$ if inequality

$$
f(t x+(1-t) y) \leq t f(x)+(1-t) f(y)
$$

holds for all $x, y \in I$ and $t \in[0,1]$. Geometrically, this means that if $P, Q$ and $R$ are three distinct points on the graph of $f$ with $Q$ between $P$ and $R$, then $Q$ is on or below the chord $P R$.

Let $f: I \subseteq \mathbb{R} \rightarrow \mathbb{R}$ be a convex function and $a, b \in I$ with $a<b$. The following double inequality:

$$
f\left(\frac{a+b}{2}\right) \leq \frac{1}{b-a} \int_{a}^{b} f(x) d x \leq \frac{f(a)+f(b)}{2}
$$

is known in the literature as Hadamard's inequality for convex function. Keep in mind that some of the classical inequalities for means can come from (1.2) for convenient particular selections of the function $f$. If $f$ is concave, this double inequality holds in the opposite way.

The inequalities (1.2) which have been frequently used in a variety of settings, has come to a significant groundwork in mathematical analysis and optimization. 
Many reports have provided new proofs, extensions and considering its refinements, generalizations, numerous interpolations and applications, for example, in the theory of special means and information theory. For some results on generalizations, extensions and applications of the Hermite-Hadamard inequalities, see [14, 15, 17], $[2-4,7,10,11,21,23,24,26],[5,6,16,18,19,22]$.

Definition 1 ([15]). We say that $f: I \rightarrow \mathbb{R}$ is Godunova-Levin function or that $f$ belongs to the class $Q(I)$ if $f$ is non-negative and for all $x, y \in I$ and $t \in(0,1)$ we have

$$
f(t x+(1-t) y) \leq \frac{f(x)}{t}+\frac{f(y)}{1-t} .
$$

Definition 2 ([14]). We say that $f: I \subseteq \mathbb{R} \rightarrow \mathbb{R}$ is a $P$-function or that $f$ belongs to the class $P(I)$ if $f$ is nonnegative and for all $x, y \in I$ and $t \in[0,1]$, we have

$$
f(t x+(1-t) y) \leq f(x)+f(y) .
$$

Definition 3 ([17]). Let $s \in(0,1]$. A function $f:(0, \infty] \rightarrow[0, \infty]$ is said to be $s$-convex in the second sense if

$$
f(t x+(1-t) y) \leq t^{s} f(x)+(1-t)^{s} f(y),
$$

for all $x, y \in(0, b]$ and $t \in[0,1]$. This class of $s$-convex functions is usually denoted by $K_{s}^{2}$.

In 1978, Breckner introduced $s$-convex functions as a generalization of convex functions in [8]. Also, in that work Breckner proved the important fact that the set valued map is $s$-convex only if the associated support function is $s$-convex function in [9]. A number of properties and connections with $s$-convex in the first sense and its generalizations are discussed in the papers $[12,13,17]$. Of course, $s$-convexity means just convexity when $s=1$.

The concept of $h$-convexity was introduced by Varošanec [26] and was generalized by Házy [16].

Definition 4 ([26]). Let $h: J \rightarrow \mathbb{R}$ be a non-negative function, $h \not \equiv 0$. We say that $f: I \rightarrow \mathbb{R}$ is an $h$-convex function, or that $f$ belongs to the class $S X(h, I)$, if $f$ is non-negative and for all $x, y \in I$ and $t \in(0,1)$ we have

$$
f(t x+(1-t) y) \leq h(t) f(x)+h(1-t) f(y) .
$$

If inequality (1.6) is reversed, then $f$ is said to be $h$-concave, i.e. $f \in S V(h, I)$. Obviously, if $h(t)=t$, then all nonnegative convex functions belong to $S X(h, I)$ and all nonnegative concave functions belong to $S V(h, I)$; if $h(t)=\frac{1}{t}$, then $S X(h, I)=$ $Q(I)$; if $h(t)=1$, then $S X(h, I) \supseteq P(I)$; and if $h(t)=t^{s}$, where $s \in(0,1)$, then $S X(h, I) \supseteq K_{s}^{2}$. 
Definition 5 ([16]). Let $X$ be a real or complex topological vector space, $D \subset X$ be a nonempty open convex set and let $h:[0,1] \rightarrow \mathbb{R}$ be a given function. We say that $f: D \rightarrow \mathbb{R}$ is an $h$-convex function if, for all $x, y \in D$ and $t \in[0,1]$, we have

$$
f(t x+(1-t) y) \leq h(t) f(x)+h(1-t) f(y) .
$$

Definition $6([22,26])$. A function $h: J \rightarrow \mathbb{R}$ is said to be a super-multiplicative function if its values are positive and satisfy

$$
h(x y) \geq h(x) h(y)
$$

for all $x, y \in J$.

If inequality (1.7) is reversed, then $h$ is said to be a sub-multiplicative function. If equality held in (1.7), then $h$ is said to be a multiplicative function.

Definition 7 ([1]). A function $h: J \rightarrow \mathbb{R}$ is said to be a super-additive function if

$$
h(x+y) \geq h(x)+h(y)
$$

for all $x, y \in J$.

Definition 8 ([25]). Two functions $h: X \rightarrow \mathbb{R}$ and $g: X \rightarrow \mathbb{R}$ are said to be similarly ordered, shortly $f$ s.o. $g$, if

$$
(f(x)-f(y))(g(x)-g(y)) \geq 0
$$

for every $x, y \in X$.

Remark 1 ([26]). Let $h$ be a non-negative function such that

$$
h(\alpha) \geq \alpha
$$

for all $\alpha \in(0,1)$. For example, the function $h_{k}(x)=x^{k}$ where $k \leq 1$ and $x>0$ has that property. If $f$ is a non-negative convex function on $I$, then for $x, y \in I$, $\alpha \in(0,1)$ we have

$$
f(\alpha x+(1-\alpha) y) \leq \alpha f(x)+(1-\alpha) f(y) \leq h(\alpha) f(x)+h(1-\alpha) f(y) .
$$

So, $f \in S X(h, I)$. Similarly, if the function $h$ has the property: $h(\alpha) \leq \alpha$ for all $\alpha \in(0,1)$, then any non-negative concave function $f$ belongs to the class $S V(h, I)$.

Proposition 1 ([26]). Let $f$ and $g$ be a similarly ordered functions on $I$, i.e.

$$
(f(x)-f(y))(g(x)-g(y)) \geq 0,
$$

for all $x, y \in I$. If $f \in S X\left(h_{1}, I\right), g \in S X\left(h_{2}, I\right)$ and $h(\alpha)+h(1-\alpha) \leq c$ for all $\alpha \in(0,1)$, where $h(t)=\max \left\{h_{1}(t), h_{2}(t)\right\}$ and $c$ is a fixed positive number, then the product $f g$ belongs to $S X(c h, I)$. If $f$ and $g$ are oppositely ordered, $f \in S V\left(h_{1}, I\right), g \in S V\left(h_{2}, I\right)$ and $h(\alpha)+h(1-\alpha) \geq c$ for all $\alpha \in(0,1)$, where $h(t)=\min \left\{h_{1}(t), h_{2}(t)\right\}$ and $c>0$, then the product fg belongs to $S V(\mathrm{ch}, I)$. 
Up until now, there are many reports on the two convex function, two $s$-convex functions, two $m$-convex functions or on the product of the $s$-convex function with an ordinary convex function. And in this study, in addition to its predecessors, the new inequalities on the product of classes of $h$-convex function will be obtained by using the elementary analysis and the applications in the special means for the obtained inequalities will be provided. In this paper we will imply $M(a, b)=f(a) g(a)+$ $f(b) g(b)$ and $N(a, b)=f(a) g(b)+f(b) g(a)$.

\section{MAin Results}

The following inequalities is well known in the literature; For $\mu \leq \lambda$ and $\kappa \leq \varepsilon$ and $\mu, \lambda, \kappa, \varepsilon \in \mathbb{R}$,

$$
\begin{aligned}
\mu \varepsilon+\lambda \kappa & \leq \mu \kappa+\lambda \varepsilon \\
\mu \kappa & \leq \lambda \varepsilon
\end{aligned}
$$

The inequality (2.1) is more useful than the inequality (2.2). In our proofs, the inequality (2.1) will be used.

Theorem 1. Let $f, g \in S X(h, I), h$ is super-multiplicative and $f, g$ be similarly ordered functions on $I$ for all $x, y \in I \subseteq \mathbb{R}$, and $(f, g)(x) \geq x$ and $h(t) \geq t$. Then for all $t \in[0,1]$, we have;

$$
\begin{aligned}
& \frac{2 a+3 b}{6}(f(a)+g(a))+\frac{3 a+2 b}{6}(f(b)+g(b)) \\
& \leq \frac{1}{b-a} \int_{a}^{b}(f g)(x) d x+(f g)(a) \int_{0}^{1}\left[h(t(1-t))+h\left(t^{2}\right)\right] d t \\
& +(f g)(b) \int_{0}^{1}\left[h(t(1-t))+h\left((1-t)^{2}\right)\right] d t .
\end{aligned}
$$

Proof. Since $f, g$ are $h$-convex functions on $I$, we have

$$
\begin{aligned}
& f(\alpha x+\beta y) \leq h(\alpha) f(x)+h(\beta) f(y) \\
& g(\alpha x+\beta y) \leq h(\alpha) g(x)+h(\beta) g(y)
\end{aligned}
$$

for all $\alpha, \beta \in(0,1), \alpha+\beta=1$. Using the elementary inequality $\mu \leq \lambda$ and $\kappa \leq \varepsilon$ then $\mu \varepsilon+\lambda \kappa \leq \mu \kappa+\lambda \varepsilon$ for $\mu, \lambda, \kappa, \varepsilon \in \mathbb{R}$, so we get

$$
\begin{aligned}
& f(\alpha x+\beta y)[h(\alpha) g(x)+h(\beta) g(y)] \\
& +g(\alpha x+\beta y)[h(\alpha) f(x)+h(\beta) f(y)] \\
& \leq f(\alpha x+\beta y) g(\alpha x+\beta y) \\
& +[h(\alpha) f(x)+h(\beta) f(y)][h(\alpha) g(x)+h(\beta) g(y)]
\end{aligned}
$$

Using the other properties of $f, g$ and $h$ in Theorem 1, we get

$$
f(\alpha x+\beta y)[h(\alpha) g(x)+h(\beta) g(y)]
$$




$$
\begin{aligned}
& +g(\alpha x+\beta y)[h(\alpha) f(x)+h(\beta) f(y)] \\
& =h(\alpha) g(x) f(\alpha x+\beta y)+h(\beta) g(y) f(\alpha x+\beta y) \\
& +h(\alpha) f(x) g(\alpha x+\beta y)+h(\beta) f(y) g(\alpha x+\beta y) .
\end{aligned}
$$

Since $f, g$ and $h$ are nonnegative functions, we write

$$
\begin{aligned}
& h(\alpha)[g(x) f(\alpha x+\beta y)+f(x) g(\alpha x+\beta y)] \\
& +h(\beta)[g(y) f(\alpha x+\beta y)+f(y) g(\alpha x+\beta y)] \\
& \geq \alpha[(\alpha x+\beta y) g(x)+(\alpha x+\beta y) f(x)] \\
& +\beta[(\alpha x+\beta y) g(y)+(\alpha x+\beta y) f(y)] \\
& =\alpha[(\alpha x+\beta y)(g(x)+f(x))]+\beta[(\alpha x+\beta y)(g(y)+f(y))] .
\end{aligned}
$$

For the right hand side of (2.4), again using the other properties of $f, g$ and $h$ in Theorem 1, we can write

$$
\begin{aligned}
& f(\alpha x+\beta y) g(\alpha x+\beta y) \\
& +[h(\alpha) f(x)+h(\beta) f(y)][h(\alpha) g(x)+h(\beta) g(y)] \\
& =f(\alpha x+\beta y) g(\alpha x+\beta y)+h^{2}(\alpha) f(x) g(x)+h^{2}(\beta) f(y) g(y) \\
& +h(\alpha) h(\beta) f(x) g(y)+h(\alpha) h(\beta) f(y) g(x) \\
& \leq f(\alpha x+\beta y) g(\alpha x+\beta y)+h^{2}(\alpha) f(x) g(x)+h^{2}(\beta) f(y) g(y) \\
& +h(\alpha \beta) f(x) g(y)+h(\alpha \beta) f(y) g(x) \\
& =f(\alpha x+\beta y) g(\alpha x+\beta y)+h^{2}(\alpha) f(x) g(x)+h^{2}(\beta) f(y) g(y) \\
& +h(\alpha \beta)(f(x) g(y)+f(y) g(x)) \\
& \leq f(\alpha x+\beta y) g(\alpha x+\beta y)+h^{2}(\alpha) f(x) g(x)+h^{2}(\beta) f(y) g(y) \\
& +h(\alpha \beta)(f(x) g(x)+f(y) g(y)) \\
& =f(\alpha x+\beta y) g(\alpha x+\beta y)+\left[h^{2}(\alpha)+h(\alpha \beta)\right] f(x) g(x) \\
& +\left[h^{2}(\beta)+h(\alpha \beta)\right] f(y) g(y)
\end{aligned}
$$

Now by combining expression (2.5) and (2.6), we obtain

$$
\begin{aligned}
& \alpha[(\alpha x+\beta y)(g(x)+f(x))]+\beta[(\alpha x+\beta y)(g(y)+f(y))] \\
& \leq f(\alpha x+\beta y) g(\alpha x+\beta y)+\left[h^{2}(\alpha)+h(\alpha \beta)\right] f(x) g(x) \\
& +\left[h^{2}(\beta)+h(\alpha \beta)\right] f(y) g(y) .
\end{aligned}
$$

If we choose $x=a, y=b$ and $\beta=1-\alpha$ in (2.7), we have

$$
\begin{aligned}
& \left(\alpha^{2} a+(1-\alpha) b\right)(f(a)+g(a))+\left(\alpha a+(1-\alpha)^{2} b\right)(f(b)+g(b)) \\
& \leq f(\alpha a+(1-\alpha) b) g(\alpha a+(1-\alpha) b)+\left[h^{2}(\alpha)+h(\alpha(1-\alpha))\right] f(a) g(a) \\
& +\left[h^{2}(1-\alpha)+h(\alpha(1-\alpha))\right] f(b) g(b) .
\end{aligned}
$$


By multiplicatively of $h$, we deduce

$$
\begin{aligned}
& \left(\alpha^{2} a+(1-\alpha) b\right)(f(a)+g(a))+\left(\alpha a+(1-\alpha)^{2} b\right)(f(b)+g(b)) \\
& \leq f(\alpha a+(1-\alpha) b) g(\alpha a+(1-\alpha) b)+\left[h\left(\alpha^{2}\right)+h\left(\alpha-\alpha^{2}\right)\right] f(a) g(a) \\
& +\left[h\left((1-\alpha)^{2}\right)+h\left(\alpha-\alpha^{2}\right)\right] f(b) g(b) .
\end{aligned}
$$

By integrating the result with respect to $\alpha$ over $[0,1]$, we obtain

$$
\begin{aligned}
& \left(a \int_{0}^{1} \alpha^{2} d \alpha+b \int_{0}^{1}(1-\alpha) d \alpha\right)(f(a)+g(a)) \\
& +\left(a \int_{0}^{1} \alpha d \alpha+b \int_{0}^{1}(1-\alpha)^{2} d \alpha\right)(f(b)+g(b)) \\
& =\frac{2 a+3 b}{6}(f(a)+g(a))+\frac{3 a+2 b}{6}(f(b)+g(b)) \\
& \leq \frac{1}{b-a} \int_{a}^{b} f g(x) d x+f(a) g(a) \int_{0}^{1}\left[h\left(\alpha^{2}\right)+h\left(\alpha-\alpha^{2}\right)\right] d \alpha \\
& +f(b) g(b) \int_{0}^{1}\left[h\left((1-\alpha)^{2}\right)+h\left(\alpha-\alpha^{2}\right)\right] d \alpha
\end{aligned}
$$

which completes the proof.

Corollary 1. If in (2.3) we take $h(t)=1$, then we get an integral inequality for $P$-functions with launching of necessary mathematical operations,

$$
\begin{aligned}
& \frac{2 a+3 b}{6}(f(a)+g(a))+\frac{3 a+2 b}{6}(f(b)+g(b)) \\
& \leq \frac{1}{b-a} \int_{a}^{b}(f g)(x) d x+2 M(a, b) .
\end{aligned}
$$

Corollary 2. If in (2.3) we take $h(t)=t$, then we get an integral inequality for ordinary convex functions with launching of necessary mathematical operations,

$$
\begin{aligned}
& \frac{2 a+3 b}{6}(f(a)+g(a))+\frac{3 a+2 b}{6}(f(b)+g(b)) \\
& \leq \frac{1}{b-a} \int_{a}^{b}(f g)(x) d x+(f g)(a) \int_{0}^{1}\left[\left(t-t^{2}\right)+\left(t^{2}\right)\right] d t \\
& +(f g)(b) \int_{0}^{1}\left[\left(t-t^{2}\right)+\left((1-t)^{2}\right)\right] d t \\
& =\frac{1}{b-a} \int_{a}^{b}(f g)(x) d x+\frac{M(a, b)}{2} .
\end{aligned}
$$


Corollary 3. If in (2.3) we take $h(t)=t^{s}$, then we obtain an integral inequality for s-convex functions in the second sense with use of the Beta function of Euler type

$$
\begin{aligned}
& \frac{2 a+3 b}{6}(f(a)+g(a))+\frac{3 a+2 b}{6}(f(b)+g(b)) \\
& \leq \frac{1}{b-a} \int_{a}^{b}(f g)(x) d x+(f g)(a) \int_{0}^{1}\left[t^{s}(1-t)^{s}+t^{2 s}\right] d t \\
& +(f g)(b) \int_{0}^{1}\left[t^{s}(1-t)^{s}+(1-t)^{2 s}\right] d t \\
& =\frac{1}{b-a} \int_{a}^{b}(f g)(x) d x+(f g)(a)[\beta(s+1, s+1)+\beta(2 s+1,1)] \\
& +(f g)(b)[\beta(s+1, s+1)+\beta(1,2 s+1)] \\
& =\frac{1}{b-a} \int_{a}^{b}(f g)(x) d x+M(a, b)[\beta(s+1, s+1)+\beta(2 s+1,1)]
\end{aligned}
$$

Theorem 2. Let $f, g \in S X(h, I), h$ is super-additive and nonnegative such that $h(\alpha) \geq \alpha$ and $f, g$ be similarly ordered functions on I for all $x, y \in I \subseteq \mathbb{R}$. Then for all $\alpha \in(0,1)$ and $\alpha+\beta=1$ we have following inequality;

$$
\begin{aligned}
& \frac{M(a, b)}{6}+\frac{N(a, b)}{3} \\
& \leq h(1)\left[f(a) g(a) \int_{0}^{1} h(\alpha) d \alpha+f(b) g(b) \int_{0}^{1} h(1-\alpha) d \alpha\right] .
\end{aligned}
$$

Proof. Since $f, g$ are $h$-convex functions on $I$, and using right hand side of (1.11), we have

$$
\begin{gathered}
\alpha f(x)+\beta f(y) \leq h(\alpha) f(x)+h(\beta) f(y) \\
\alpha g(x)+\beta g(y) \leq h(\alpha) g(x)+h(\beta) g(y)
\end{gathered}
$$

for all $\alpha, \beta \in(0,1), \alpha+\beta=1$. Using the elementary inequality $\mu \leq \lambda$ and $\kappa \leq \varepsilon$ then $\mu \varepsilon+\lambda \kappa \leq \mu \kappa+\lambda \varepsilon$ for $\mu, \lambda, \kappa, \varepsilon \in \mathbb{R}$, so we get

$$
\begin{aligned}
& {[\alpha f(x)+\beta f(y)][h(\alpha) g(x)+h(\beta) g(y)]} \\
& +[\alpha g(x)+\beta g(y)][h(\alpha) f(x)+h(\beta) f(y)] \\
& \leq[\alpha f(x)+\beta f(y)][\alpha g(x)+\beta g(y)] \\
& +[h(\alpha) f(x)+h(\beta) f(y)][h(\alpha) g(x)+h(\beta) g(y)] .
\end{aligned}
$$

Using the other properties of $h$ in Theorem 2 on the left hand side of (2.9), we get

$$
\begin{aligned}
& {[\alpha f(x)+\beta f(y)][h(\alpha) g(x)+h(\beta) g(y)]} \\
& +[\alpha g(x)+\beta g(y)][h(\alpha) f(x)+h(\beta) f(y)] \\
& =\alpha h(\alpha) f(x) g(x)+\alpha h(\beta) f(x) g(y)
\end{aligned}
$$




$$
\begin{aligned}
& +\beta h(\alpha) f(y) g(x)+\beta h(\beta) f(y) g(y) \\
& +\alpha h(\alpha) f(x) g(x)+\alpha h(\beta) f(y) g(x) \\
& +\beta h(\alpha) f(x) g(y)+\beta h(\beta) f(y) g(y) \\
& =2 \alpha h(\alpha) f(x) g(x)+\alpha h(\beta)[f(x) g(y)+f(y) g(x)] \\
& +\beta h(\alpha)[f(x) g(y)+f(y) g(x)] \\
& +2 \beta h(\beta) f(y) g(y),
\end{aligned}
$$

if $h$ be a non-negative function that $h(\alpha) \geq \alpha$

$$
\begin{aligned}
& 2 \alpha h(\alpha) f(x) g(x)+2 \beta h(\beta) f(y) g(y) \\
& +[\alpha h(\beta)+\beta h(\alpha)][f(x) g(y)+f(y) g(x)] \\
& \geq 2 \alpha^{2} f(x) g(x)+2 \alpha \beta[f(x) g(y)+f(y) g(x)]+2 \beta^{2} f(y) g(y) .
\end{aligned}
$$

However, using the other properties of $f, g$ and $h$ in Theorem 2 on the right hand side of (2.9), we get

$$
\begin{aligned}
& {[\alpha f(x)+\beta f(y)][\alpha g(x)+\beta g(y)]} \\
& +[h(\alpha) f(x)+h(\beta) f(y)][h(\alpha) g(x)+h(\beta) g(y)] \\
& =\alpha^{2} f(x) g(x)+\alpha \beta f(x) g(y) \\
& +\alpha \beta f(y) g(x)+\beta^{2} f(y) g(y) \\
& +h^{2}(\alpha) f(x) g(x)+h(\alpha) h(\beta) f(x) g(y) \\
& +h(\alpha) h(\beta) f(y) g(x)+h^{2}(\beta) f(y) g(y) \\
& =\alpha^{2} f(x) g(x)+\beta^{2} f(y) g(y) \\
& +\alpha \beta[f(x) g(y)+f(y) g(x)] \\
& +h^{2}(\alpha) f(x) g(x)+h^{2}(\beta) f(y) g(y) \\
& +h(\alpha) h(\beta)[f(x) g(y)+f(y) g(x)] \\
& \leq \alpha^{2} f(x) g(x)+\beta^{2} f(y) g(y)+\alpha \beta[f(x) g(x)+f(y) g(y)] \\
& +h^{2}(\alpha) f(x) g(x)+h^{2}(\beta) f(y) g(y) \\
& +h(\alpha) h(\beta)[f(x) g(x)+f(y) g(y)] \\
& =\left[\alpha^{2}+\alpha \beta+h^{2}(\alpha)+h(\alpha) h(\beta)\right] f(x) g(x) \\
& +\left[\alpha \beta+\beta^{2}+h(\alpha) h(\beta)+h^{2}(\beta)\right] f(y) g(y) \\
& \leq\left[\alpha^{2}+\alpha \beta+h(\alpha) h(\alpha+\beta)\right] f(x) g(x) \\
& +\left[\alpha \beta+\beta^{2}+h(\beta) h(\alpha+\beta)\right] f(y) g(y) \\
& =\left[\alpha^{2}+\alpha \beta+h(\alpha) h(1)\right] f(x) g(x) \\
& +\left[\alpha \beta+\beta^{2}+h(\beta) h(1)\right] f(y) g(y)
\end{aligned}
$$


Then by expression (2.10) and (2.11);

$$
\begin{aligned}
& \alpha^{2} f(x) g(x)+2 \alpha \beta[f(x) g(y)+f(y) g(x)]+\beta^{2} f(y) g(y) \\
& \leq[\alpha \beta+h(\alpha) h(1)] f(x) g(x)+[\alpha \beta+h(\beta) h(1)] f(y) g(y)
\end{aligned}
$$

by taking $x=a, y=b$ and $\beta=1-\alpha$ in (2.12), we have

$$
\begin{aligned}
& \alpha^{2} f(a) g(a)+2 \alpha(1-\alpha)[f(a) g(b)+f(b) g(a)]+(1-\alpha)^{2} f(b) g(b) \\
& \leq[\alpha(1-\alpha)+h(\alpha) h(1)] f(a) g(a)+[\alpha(1-\alpha)+h(1-\alpha) h(1)] f(b) g(b)
\end{aligned}
$$

By integrating the result with respect to $\alpha$ over $[0,1]$, we get

$$
\begin{aligned}
& f(a) g(a) \int_{0}^{1} \alpha^{2} d \alpha+2[f(a) g(b)+f(b) g(a)] \int_{0}^{1} \alpha(1-\alpha) d \alpha \\
& +f(b) g(b) \int_{0}^{1}(1-\alpha)^{2} d \alpha \\
& =\frac{f(a) g(a)+f(b) g(b)}{3}+\frac{f(a) g(b)+f(b) g(a)}{3} \\
& \leq f(a) g(a)\left[\int_{0}^{1} \alpha(1-\alpha) d \alpha+h(1) \int_{0}^{1} h(\alpha) d \alpha\right] \\
& +f(b) g(b)\left[\int_{0}^{1} \alpha(1-\alpha) d \alpha+h(1) \int_{0}^{1} h(1-\alpha) d t\right] \\
& =\frac{f(a) g(a)+f(b) g(b)}{6} \\
& +h(1)\left[f(a) g(a) \int_{0}^{1} h(\alpha) d \alpha+f(b) g(b) \int_{0}^{1} h(1-\alpha) d \alpha\right]
\end{aligned}
$$

which completes the proof of (2.8).

Corollary 4. In Theorem 2, if we choose $h(t)=t$, then inequality of (2.8) brings inequality (1.12) down.

Corollary 5. In Theorem 2, if we choose $h(t)=t^{s}$, then we get an integral inequality for $s$-convex functions in the second sense;

$$
\frac{M(a, b)}{6}+\frac{N(a, b)}{3} \leq \frac{M(a, b)}{s+1} .
$$

And, in (2.13), if we choose $s=1$, then the inequality of (2.13) above brings inequality (1.12) down.

Theorem 3. Let $f, g \in S X(h, I), h$ is super-additive and nonnegative such that $h(\alpha) \geq \alpha$ and $f, g$ be similarly ordered functions on I for all $x, y \in I \subseteq \mathbb{R}$. Then for all $\alpha \in(0,1)$ and $\alpha+\beta=1$, we have

$$
\frac{M(a, b)}{3}+\frac{N(a, b)}{6}
$$




$$
\leq h(1)\left[f(a) g(a) \int_{0}^{1} h(\alpha) d \alpha+f(b) g(b) \int_{0}^{1} h(1-\alpha) d \alpha\right] .
$$

Proof. As in the proof of the inequality (2.8), since $f, g$ are $h$-convex functions on $I$, and using right hand side of (1.12), we have

$$
\begin{aligned}
\alpha f(x)+\beta f(y) & \leq h(\alpha) f(x)+h(\beta) f(y) \\
\alpha g(x)+\beta g(y) & \leq h(\alpha) g(x)+h(\beta) g(y)
\end{aligned}
$$

for all $\alpha, \beta \in(0,1), \alpha+\beta=1$. Using the elementary inequality $\mu \leq \lambda$ and $\kappa \leq \varepsilon$ then $\mu \varepsilon+\lambda \kappa \leq \mu \kappa+\lambda \varepsilon$ for $\mu, \lambda, \kappa, \varepsilon \in \mathbb{R}$, so we obtain

$$
\begin{aligned}
& {[\alpha f(x)+\beta f(y)][h(\alpha) g(x)+h(\beta) g(y)]} \\
& +[\alpha g(x)+\beta g(y)][h(\alpha) f(x)+h(\beta) f(y)] \\
& \leq[\alpha f(x)+\beta f(y)][\alpha g(x)+\beta g(y)] \\
& +[h(\alpha) f(x)+h(\beta) f(y)][h(\alpha) g(x)+h(\beta) g(y)] .
\end{aligned}
$$

Using the other properties of $h$ in Theorem 3 on the left hand side of (2.15), we get

$$
\begin{aligned}
& {[\alpha f(x)+\beta f(y)][h(\alpha) g(x)+h(\beta) g(y)]} \\
& +[\alpha g(x)+\beta g(y)][h(\alpha) f(x)+h(\beta) f(y)] \\
& \geq 2 \alpha^{2} f(x) g(x)+2 \alpha \beta[f(x) g(y)+f(y) g(x)]+2 \beta^{2} f(y) g(y)
\end{aligned}
$$

However, using the other properties of $f, g$ and $h$ in Theorem 3 on the right hand side of (2.15), we obtain

$$
\begin{aligned}
& {[\alpha f(x)+\beta f(y)][\alpha g(x)+\beta g(y)]} \\
& +[h(\alpha) f(x)+h(\beta) f(y)][h(\alpha) g(x)+h(\beta) g(y)] \\
& =\alpha^{2} f(x) g(x)+\beta^{2} f(y) g(y)+\alpha \beta[f(x) g(y)+f(y) g(x)] \\
& +h^{2}(\alpha) f(x) g(x)+h^{2}(\beta) f(y) g(y)+h(\alpha) h(\beta)[f(x) g(y)+f(y) g(x)] \\
& \leq \alpha^{2} f(x) g(x)+\beta^{2} f(y) g(y)+\alpha \beta[f(x) g(x)+f(y) g(y)] \\
& +h^{2}(\alpha) f(x) g(x)+h^{2}(\beta) f(y) g(y)+h(\alpha) h(\beta)[f(x) g(x)+f(y) g(y)] \\
& \leq h^{2}(\alpha) f(x) g(x)+h^{2}(\beta) f(y) g(y)+h(\alpha) h(\beta)[f(x) g(x)+f(y) g(y)] \\
& +h^{2}(\alpha) f(x) g(x)+h^{2}(\beta) f(y) g(y)+h(\alpha) h(\beta)[f(x) g(x)+f(y) g(y)] \\
& =2\left(h^{2}(\alpha)+h(\alpha) h(\beta)\right) f(x) g(x)+2\left(h^{2}(\beta)+h(\alpha) h(\beta)\right) f(y) g(y) \\
& \leq 2 h(\alpha) h(\alpha+\beta) f(x) g(x)+2 h(\beta) h(\alpha+\beta) f(y) g(y) \\
& =2 h(\alpha+\beta)[h(\alpha) f(x) g(x)+h(\beta) f(y) g(y)] \\
& =2 h(1)[h(\alpha) f(x) g(x)+h(\beta) f(y) g(y)]
\end{aligned}
$$

Then by expression (2.16) and (2.17);

$$
\alpha^{2} f(x) g(x)+\alpha \beta[f(x) g(y)+f(y) g(x)]+\beta^{2} f(y) g(y)
$$




$$
\leq h(1)[h(\alpha) f(x) g(x)+h(\beta) f(y) g(y)]
$$

by taking $x=a, y=b$ and $\beta=1-\alpha$ in (2.18), we have

$$
\begin{aligned}
& t^{2} f(a) g(a)+t(1-t)[f(a) g(b)+f(b) g(a)]+(1-t)^{2} f(b) g(b) \\
& \leq h(1)[h(\alpha) f(a) g(a)+h(1-\alpha) f(b) g(b)] .
\end{aligned}
$$

By integrating the result with respect to $\alpha$ over $[0,1]$, we obtain

$$
\begin{aligned}
& f(a) g(a) \int_{0}^{1} \alpha^{2} d \alpha+2[f(a) g(b)+f(b) g(a)] \int_{0}^{1} \alpha(1-\alpha) d \alpha \\
& +f(b) g(b) \int_{0}^{1}(1-\alpha)^{2} d \alpha \\
& =\frac{f(a) g(a)+f(b) g(b)}{3}+\frac{f(a) g(b)+f(b) g(a)}{6} \\
& \leq h(1)[h(\alpha) f(a) g(a)+h(1-\alpha) f(b) g(b)] \\
& =h(1)\left[f(a) g(a) \int_{0}^{1} h(\alpha) d \alpha+f(b) g(b) \int_{0}^{1} h(1-\alpha) d \alpha\right]
\end{aligned}
$$

which completes the proof of (2.14).

Theorem 4. Let $f, g \in S X(h, I), h$ is super-additive. Then for all $\alpha \in(0,1)$, we have following inequality;

$$
\begin{aligned}
& {\left[f\left(\frac{a+b}{2}\right) \frac{g(a)+g(b)}{2}+g\left(\frac{a+b}{2}\right) \frac{f(a)+f(b)}{2}\right]} \\
& \times\left[\int_{0}^{1} h(\alpha) d \alpha+\int_{0}^{1} h(1-\alpha) d \alpha\right] \\
& \leq(f g)\left(\frac{a+b}{2}\right)+h^{2}(1)(M(a, b)+N(a, b)) .
\end{aligned}
$$

Proof. Since $f, g \in S X(h, I)$, we have

$$
\begin{aligned}
f\left(\frac{a+b}{2}\right) & =f\left(\frac{\alpha a+(1-\alpha) b}{2}+\frac{(1-\alpha) a+\alpha b}{2}\right) \\
& \leq \frac{f(\alpha a+(1-\alpha) b)+f((1-\alpha) a+\alpha b)}{2} \\
& \leq \frac{h(\alpha) f(a)+h(1-\alpha) f(b)+h(1-\alpha) f(a)+h(\alpha) f(b)}{2} \\
& =\frac{[h(\alpha)+h(1-\alpha)][f(a)+f(b)]}{2} \\
g\left(\frac{a+b}{2}\right) & \leq \frac{[h(\alpha)+h(1-\alpha)][g(a)+g(b)]}{2}
\end{aligned}
$$


for all $\alpha \in(0,1)$. Using the elementary inequality $\mu \leq \lambda$ and $\kappa \leq \varepsilon$ then $\mu \varepsilon+\lambda \kappa \leq$ $\mu \kappa+\lambda \varepsilon$ for $\mu, \lambda, \kappa, \varepsilon \in \mathbb{R}$, so we get

$$
\begin{aligned}
& f\left(\frac{a+b}{2}\right) \frac{[h(\alpha)+h(1-\alpha)][g(a)+g(b)]}{2} \\
& +g\left(\frac{a+b}{2}\right) \frac{[h(\alpha)+h(1-\alpha)][f(a)+f(b)]}{2} \\
& =\left[f\left(\frac{a+b}{2}\right) \frac{g(a)+g(b)}{2}+g\left(\frac{a+b}{2}\right) \frac{f(a)+f(b)}{2}\right][h(\alpha)+h(1-\alpha)] \\
& \leq f\left(\frac{a+b}{2}\right) g\left(\frac{a+b}{2}\right)+\frac{[h(\alpha)+h(1-\alpha)]^{2}[f(a)+f(b)][g(a)+g(b)]}{4} \\
& \leq f\left(\frac{a+b}{2}\right) g\left(\frac{a+b}{2}\right)+\frac{[h(1)]^{2}[f(a)+f(b)][g(a)+g(b)]}{4},
\end{aligned}
$$

by integrating the result with respect to $\alpha$ over [0,1], we obtain (2.19).

Theorem 5. Let $f, g \in S X(h, I)$, and $h$ be super-multiplicative. Then for all $\alpha \in(0,1)$, we have following inequality;

$$
\begin{aligned}
& \frac{2 f\left(\frac{a+b}{2}\right) g\left(\frac{a+b}{2}\right)}{M(a, b)} \\
& \leq\left[\int_{0}^{1} h\left(\alpha^{2}\right) d \alpha+2 \int_{0}^{1} h\left(\alpha-\alpha^{2}\right) d \alpha+\int_{0}^{1} h\left((1-\alpha)^{2}\right) d \alpha\right] .
\end{aligned}
$$

Proof. Since $f, g \in S X(h, I)$, we write

$$
\begin{aligned}
& f\left(\frac{a+b}{2}\right) \leq[h(\alpha)+h(1-\alpha)] \frac{f(a)+f(b)}{2} \\
& g\left(\frac{a+b}{2}\right) \leq[h(\alpha)+h(1-\alpha)] \frac{g(a)+g(b)}{2}
\end{aligned}
$$

for all $\alpha \in(0,1)$. Since $h$ is super-multiplicative function and $f$ and $g$ are similarly ordered functions, we get

$$
\begin{aligned}
& f\left(\frac{a+b}{2}\right) g\left(\frac{a+b}{2}\right) \\
& \leq \frac{[h(\alpha)+h(1-\alpha)]^{2}}{4}(f(a)+f(b))(g(a)+g(b)) \\
& =\frac{\left[h^{2}(\alpha)+2 h(\alpha) h(1-\alpha)+h^{2}(1-\alpha)\right]^{2}}{4} \\
& \mathrm{x}(f(a) g(a)+f(b) g(b)+f(a) g(b)+f(b) g(a))
\end{aligned}
$$




$$
\leq \frac{\left[h\left(\alpha^{2}\right)+2 h(\alpha(1-\alpha))+h\left((1-\alpha)^{2}\right)\right]^{2}(f(a) g(a)+f(b) g(b))}{2}
$$

by integrating the result with respect to $\alpha$ over [0,1], we obtain (2.20).

Corollary 6. If in (2.20) we take $h(\alpha)=1$, then we obtain an integral inequality for P-functions with launching of necessary mathematical operations,

$$
f\left(\frac{a+b}{2}\right) g\left(\frac{a+b}{2}\right) \leq 2 M(a, b)
$$

Corollary 7. If in (2.20) we take $h(\alpha)=\alpha$, then we obtain an integral inequality for ordinary convex functions with launching of necessary mathematical operations,

$$
f\left(\frac{a+b}{2}\right) g\left(\frac{a+b}{2}\right) \leq \frac{M(a, b)}{2} .
$$

Corollary 8. If in (2.20) we take $h(\alpha)=\alpha^{s}$, then we obtain an integral inequality for s-convex functions in the second sense with the use of the Beta function of Euler type

$$
\begin{aligned}
& f\left(\frac{a+b}{2}\right) g\left(\frac{a+b}{2}\right) \\
& \leq \frac{M(a, b)}{2}\left[\int_{0}^{1} \alpha^{2 s} d \alpha+2 \int_{0}^{1} \alpha^{s}(1-\alpha)^{s} d \alpha+\int_{0}^{1}(1-\alpha)^{2 s} d \alpha\right] \\
& =\frac{M(a, b)}{2}\{\beta(2 s+1,1)+2 \beta(2 s+1,2 s+1)+\beta(1,2 s+1)\} \\
& =M(a, b)\{\beta(2 s+1,1)+\beta(2 s+1,2 s+1)\} .
\end{aligned}
$$

Theorem 6. Let $I=[a, b] \subseteq \mathbb{R}, f, g \in S X(h, I), h$ is super-multiplicative and $f$, $g$ are symmetric about $\frac{a+b}{2}$, then for all $x \in I$ ad $\alpha \in[0,1]$, we have the following inequalities;

$$
\begin{aligned}
& \frac{1}{b-a} \int_{a}^{b} f(x) g(x) d x \\
& \leq \frac{M(a, b)+N(a, b)}{4} \\
& \times\left[\int_{0}^{1} h\left(\alpha^{2}\right) d \alpha+2 \int_{0}^{1} h(\alpha(1-\alpha)) d \alpha+\int_{0}^{1} h\left((1-\alpha)^{2}\right) d \alpha\right]
\end{aligned}
$$

Proof. Since $f, g \in S X(h, I)$, we can write

$$
\begin{aligned}
f(\alpha a+(1-\alpha) b)+f((1-\alpha) a+\alpha b) & \leq[h(\alpha)+h(1-\alpha)][f(a)+f(b)] \\
g(\alpha a+(1-\alpha) b)+g((1-\alpha) a+\alpha b) & \leq[h(\alpha)+h(1-\alpha)][g(a)+g(b)]
\end{aligned}
$$


for all $\alpha \in[0,1]$. Since $h$ is a super-multiplicative function, and $f$ and $g$ are symmetric about $\frac{a+b}{2}$, we get

$$
\begin{aligned}
& {[f(\alpha a+(1-\alpha) b)+f((1-\alpha) a+\alpha b)][g(\alpha a+(1-\alpha) b)+g((1-\alpha) a+\alpha b)]} \\
& =4 f(\alpha a+(1-\alpha) b) g(\alpha a+(1-\alpha) b) \\
& \leq[h(\alpha)+h(1-\alpha)]^{2}[f(a)+f(b)][g(a)+g(b)] \\
& =\left[h^{2}(\alpha)+2 h(\alpha) h(1-\alpha)+h^{2}(1-\alpha)\right][M(a, b)+N(a, b)] \\
& \leq\left[h\left(\alpha^{2}\right)+2 h(\alpha(1-\alpha))+h\left((1-\alpha)^{2}\right)\right][M(a, b)+N(a, b)] .
\end{aligned}
$$

By integrating the result with respect to $\alpha$ over $[0,1]$, and taking into account the change of variable $x=\alpha a+(1-\alpha) b$, we obtain

$$
\begin{aligned}
& \frac{4}{b-a} \int_{a}^{b} f(x) g(x) d x \\
& \leq[M(a, b)+N(a, b)]\left[\int_{0}^{1} h\left(\alpha^{2}\right) d \alpha+2 \int_{0}^{1} h(\alpha(1-\alpha)) d \alpha+\int_{0}^{1} h\left((1-\alpha)^{2}\right) d \alpha\right]
\end{aligned}
$$

which completes the proof.

Corollary 9. If in (2.21) we take $h(\alpha)=1$, then we obtain an integral inequality for P-functions with launching of necessary mathematical operations,

$$
\frac{1}{b-a} \int_{a}^{b} f(x) g(x) d x \leq M(a, b)+N(a, b) .
$$

Corollary 10. If in (2.21) we take $h(\alpha)=\alpha$, then we obtain an integral inequality for ordinary convex functions with the use of the necessary mathematical operations,

$$
\frac{1}{b-a} \int_{a}^{b} f(x) g(x) d x \leq \frac{M(a, b)+N(a, b)}{4} .
$$

Corollary 11. If in (2.21) we take $h(\alpha)=\alpha^{s}$, then we obtain an integral inequality for s-convex functions in the second sense the with use of the Beta function of Euler type

$$
\frac{1}{b-a} \int_{a}^{b} f(x) g(x) d x \leq \frac{M(a, b)+N(a, b)}{2}\{\beta(2 s+1,1)+\beta(2 s+1,2 s+1)\} .
$$

\section{Applications to Some Special Means}

We now consider the applications of our Theorems to the following special means

The arithmetic mean: $A=A(a, b):=\frac{a+b}{2}, a, b \geq 0$,

The geometric mean: $G=G(a, b):=\sqrt{a b}, a, b \geq 0$,

The quadratic mean: $K=K(a, b):=\sqrt{\frac{a^{2}+b^{2}}{2}} a, b \geq 0$, 
The p-logarithmic mean: $L_{p}=L_{p}(a, b):=\left\{\begin{array}{ll}{\left[\frac{b^{p+1}-a^{p+1}}{(p+1)(b-a)}\right]^{1 / p}} & \text { if } a \neq b \\ a & \text { if } a=b\end{array}\right.$, $p \in \mathbb{R} \backslash\{-1,0\} ; a, b>0$.

The following inequality is well known in the literature:

$$
H \leq G \leq L \leq I \leq A \leq K
$$

It is also known that $L_{p}$ is monotonically increasing over $p \in \mathbb{R}$, denoting $L_{1}=A$, $L_{0}=I$ and $L_{-1}=L$.

The following propositions hold:

Proposition 2. Let $a, b \in \mathbb{R}, 0<a<b$ and $n \in \mathbb{Z},|n| \geq 1$. Then we have:

$$
\begin{aligned}
& \frac{4}{3} A\left(a^{n+1}, b^{n+1}\right)+2 G^{2}(a, b) A\left(a^{n-1}, b^{n-1}\right) \\
& \leq L_{2 n}^{2 n}(a, b)+A\left(a^{2 n}, b^{2 n}\right) .
\end{aligned}
$$

Proof. If we apply Theorem 1 for $f(x)=g(x)=x^{n}, h(\alpha)=\alpha$ where $x \in \mathbb{R}$, $n \in \mathbb{Z},|n| \geq 1$, we get the proof (3.1).

Proposition 3. Let $a, b \in \mathbb{R}, 0<a<b$ and $n \in \mathbb{Z},|n| \geq 1$. Then we have:

$$
G^{2}\left(a^{n}, b^{n}\right) \leq 2 A\left(a^{2 n}, b^{2 n}\right) .
$$

Proof. The proof is immediate from Theorem 2 applied for $f(x)=g(x)=x^{n}$, $h(\alpha)=\alpha$ where $x \in \mathbb{R}, n \in \mathbb{Z},|n| \geq 1$.

Proposition 4. Let $a, b \in \mathbb{R}, 0<a<b$. Then we have:

$$
\frac{1}{G^{2}(a, b)} \leq K(a, b) .
$$

Proof. The assertion follows from Theorem 2 applied to $f(x)=g(x)=\frac{1}{x}, x \in$ $[a, b]$ and $h(\alpha)=\alpha$.

Proposition 5. Let $a, b \in \mathbb{R}, 0<a<b$ and $n \in \mathbb{Z},|n| \geq 1$. Then we have:

$$
G^{2}\left(a^{n}, b^{n}\right) \leq A\left(a^{2 n}, b^{2 n}\right)
$$

Proof. The assertion follows from Theorem 3 applied to $f(x)=g(x)=\frac{1}{x}, x \in$ $[a, b]$ and $h(\alpha)=\alpha$.

Proposition 6. Let $a, b \in \mathbb{R}, 0<a<b$ and $n \in \mathbb{Z},|n| \geq 1$. Then we have:

$$
L_{2 n}^{2 n}(a, b) \leq \frac{A\left(a^{2 n}, b^{2 n}\right)+G^{2}\left(a^{n}, b^{n}\right)}{2} .
$$

Proof. If we apply Theorem 6 for $f(x)=g(x)=x^{n}, h(\alpha)=\alpha$ where $x \in \mathbb{R}$, $n \in \mathbb{Z},|n| \geq 1$, we get the proof (3.5). 
Proposition 7. Let $a, b \in \mathbb{R}, 0<a<b$ and $n \in \mathbb{Z},|n| \geq 1$. Then we have:

$$
G(a, b) \leq A(a, b)
$$

Proof. If we apply Theorem 6 for $f(x)=g(x)=\frac{1}{x}, x \in[a, b]$ and $h(\alpha)=\alpha$, we get the proof (3.6).

\section{REFERENCES}

[1] H. Alzer, "A superadditive property of Hadamard's gamma function," Abh. Math. Semin. Univ. Hamb., vol. 79, no. 1, pp. 11-23, 2009.

[2] M. Bessenyei, "Hermite-Hadamard-type inequalities for generalized convex functions," JIPAM, J. Inequal. Pure Appl. Math., vol. 9, no. 3, p. 51, 2008.

[3] M. Bessenyei, "The Hermite-Hadamard inequality on simplices," Am. Math. Mon., vol. 115, no. 4, pp. 339-345, 2008.

[4] M. Bessenyei, "The Hermite-Hadamard inequality in Beckenbach's setting," J. Math. Anal. Appl., vol. 364, no. 2, pp. 366-383, 2010.

[5] M. Bessenyei and Z. Páles, "Higher-order generalizations of Hadamard's inequality," Publ. Math., vol. 61, no. 3-4, pp. 623-643, 2002.

[6] M. Bessenyei and Z. Páles, "Characterizations of convexity via Hadamard's inequality," Math. Inequal. Appl., vol. 9, no. 1, pp. 53-62, 2006.

[7] M. Bombardelli and S. Varošanec, "Properties of $h$-convex functions related to the HermiteHadamard-Fejér inequalities,” Comput. Math. Appl., vol. 58, no. 9, pp. 1869-1877, 2009.

[8] W. W. Breckner, "Stetigkeitsaussagen für eine Klasse verallgemeinerter konvexer Funktionen in topologischen linearen Räumen,” Publ. Inst. Math., Nouv. Sér., vol. 23, pp. 13-20, 1978.

[9] W. W. Breckner, "Continuity of generalized convex and generalized concave set-valued functions," Rev. Anal. Numér. Théor. Approx., vol. 22, no. 1, pp. 39-51, 1993.

[10] P. Burai and A. Hazy, "On Orlicz-convex functions," in Proceedings of the 12th symposium of mathematics and its applications, "Politehnica" University of Timişoara, Timişoara, Romania, November 5-7, 2009. Timişoara: Editura Politehnica, 2010, pp. 73-78.

[11] P. Burai and A. Házy, "On approximately $h$-convex functions," J. Convex Anal., vol. 18, no. 2, pp. 447-454, 2011.

[12] P. Burai, A. Házy, and T. Juhász, "Bernstein-Doetsch type results for $s$-convex functions," Publ. Math., vol. 75, no. 1-2, pp. 23-31, 2009.

[13] P. Burai, A. Házy, and T. Juhász, "On approximately Breckner $s$-convex functions," Control Cybernet., vol. 40, no. 1, pp. 91-99, 2011.

[14] S. S. Dragomir, J. E. Pečarić, and L. E. Persson, "Some inequalities of Hadamard type," Soochow J. Math., vol. 21, no. 3, pp. 335-341, 1995.

[15] E. K. Godunova and V. I. Levin, "Neravenstva dlja funkcii sirokogo klassa, soderzascego vypuklye, monotonnye i nekotorye drugie vidy funkii," Vycislitel. Mat. i. Fiz. Mezvuzov. Sb. Nauc. Trudov, MGPI, Moskva, pp. 138-142, 1985.

[16] A. Házy, "Bernstein-Doetsch type results for $h$-convex functions," Math. Inequal. Appl., vol. 14, no. 3, pp. 499-508, 2011.

[17] H. Hudzik and L. Maligranda, "Some remarks on s-convex functions," Aequationes Math., vol. 48, no. 1, pp. 100-111, 1994.

[18] J. Makó and Z. Páles, "Implications between approximate convexity properties and approximate Hermite-Hadamard inequalities," Cent. Eur. J. Math., vol. 10, no. 3, pp. 1017-1041, 2012.

[19] J. Makó and Z. Páles, "Approximate Hermite-Hadamard type inequalities for approximately convex functions," Math. Inequal. Appl., vol. 16, no. 2, pp. 507-526, 2013. 
[20] D. S. Mitrinović, J. E. Pečarić, and A. M. Fink, Classical and new inequalities in analysis, ser. Mathematics and Its Applications. East European Series. Dordrecht: Kluwer Academic Publishers, 1993, vol. 61.

[21] M. E. Özdemir, M. Gürbüz, and A. O. Akdemir, "Inequalities for $h$-convex functions via further properties," RGMIA Research Report Collection, vol. 14, article 22, 2011.

[22] B. G. Pachpatte, Mathematical inequalities, ser. North-Holland Mathematical Library. Amsterdam: Elsevier, 2005, vol. 67.

[23] M. Z. Sarikaya, A. Saglam, and H. Yildirim, "On some Hadamard-type inequalities for $h$-convex functions," J. Math. Inequal., vol. 2, no. 3, pp. 335-341, 2008.

[24] M. Z. Sarikaya, E. Set, and M. E. Ozdemir, "On some new inequalities of Hadamard type involving h-convex functions," Acta Math. Univ. Comen., New Ser., vol. 79, no. 2, pp. 265-272, 2010.

[25] H. J. Skala, "On the characterization of certain similarly ordered super-additive functionals," Proc. Am. Math. Soc., vol. 126, no. 5, pp. 1349-1353, 1998.

[26] S. Varošanec, “On h-convexity,” J. Math. Anal. Appl., vol. 326, no. 1, pp. 303-311, 2007.

\section{Author's address}

\section{Mevlüt Tunç}

Kilis 7 Aralık University, Faculty of Science and Arts, Department of Mathematics, Kilis, 79000, Turkey.

E-mail address: mevluttunc@kilis.edu.tr 